\title{
Erratum to: In-depth Characterization via Complementing Culture-Independent Approaches of the Microbial Community in an Acidic Hot Spring of the Colombian Andes
}

\author{
Laura C. Bohorquez • Luisa Delgado-Serrano • Gina López • César Osorio-Forero • \\ Vanja Klepac-Ceraj • Roberto Kolter • Howard Junca • Sandra Baena • \\ María Mercedes Zambrano
}

Published online: 12 November 2011

(C) Springer Science+Business Media, LLC 2011

\section{Erratum to: Microb Ecol \\ DOI 10.1007/s00248-011-9943-3}

The original version of this article unfortunately contained a mistake found under the Materials and Methods section for the PCR protocol under section Pyrosequencing of V5V6 Hypervariable Regions", what says:

"PCR amplifications were done as reported at the time [29] in a $25-\mu \mathrm{L}$ reaction volume containing $2 \mu \mathrm{l}(20 \mathrm{ng})$ DNA, $0.75 \mu \mathrm{M}$ of each primer $807 \mathrm{~F}$ and $1050 \mathrm{R}$ designed by us (Table 1), $2.5 \mathrm{U}$ Pfu Turbo ${ }^{\circledR}$ DNA polymerase (Stratagene, Inc., La Jolla, CA, USA), 1× Pfu reaction buffer, $0.6 \mathrm{mM}$ dNTPs, $5 \% v / v$ dimethyl sulfoxide using the following PCR conditions: $2 \mathrm{~min}$ at $95^{\circ} \mathrm{C}, 30$ cycles consisting of denaturation for $30 \mathrm{~s}$ at $95^{\circ} \mathrm{C}$, a temperature touch down from 60 to $51^{\circ} \mathrm{C}\left(2^{\circ} \mathrm{C}\right.$ every six cycles $), 72^{\circ} \mathrm{C}$ for $1 \mathrm{~min}$ and a final extension of $72^{\circ} \mathrm{C}$ for 5 min."

The correct wording should be:

"PCR amplifications were done as reported at the time [29] in a $25-\mu \mathrm{L}$ reaction volume containing $2 \mu \mathrm{l}(20 \mathrm{ng}) \mathrm{DNA}$, $0.75 \mu \mathrm{M}$ of each primer $807 \mathrm{~F}$ and $1050 \mathrm{R}$ designed by us (Table 1), 2.5 U Pfu Turbo ${ }^{\circledR}$ DNA polymerase (Stratagene, Inc., La Jolla, CA, USA), 1× Pfu reaction buffer, 0.6 $\mathrm{mM}$ dNTPs, $5 \% v / v$ dimethyl sulfoxide using the following PCR conditions: $2 \mathrm{~min}$ at $95^{\circ} \mathrm{C}, 30$ cycles consisting of denaturation for $30 \mathrm{~s}$ at $95^{\circ} \mathrm{C}$, annealing temperature starting at $60^{\circ} \mathrm{C}$ and reducing $0.2^{\circ} \mathrm{C}$ at every cycle, elongation at $72^{\circ} \mathrm{C}$ for $1 \mathrm{~min}$ and a final extension of $72^{\circ} \mathrm{C}$ for $5 \mathrm{~min}$."

The online version of the original article can be found at http://dx.doi. org/10.1007/s00248-011-9943-3.

L. C. Bohorquez · L. Delgado-Serrano · C. Osorio-Forero •

H. Junca $\cdot$ M. M. Zambrano $(\bowtie)$

Molecular Genetics \& Biotechnology, Corporación CorpoGen,

Carrera 5 No. 66A-34,

110231, Bogotá, D.C., Colombia

e-mail: mzambrano@corpogen.org

L. C. Bohorquez $\cdot$ L. Delgado-Serrano $\cdot$ G. López $\cdot$

C. Osorio-Forero $\cdot$ H. Junca $\cdot$ S. Baena $\cdot$ M. M. Zambrano

Colombian Center for Genomics and Bioinformatics of Extreme

Environments - GeBiX,

Carrera 5 No. 66A-34,

110231, Bogotá, DC, Colombia

G. López $\cdot \mathrm{S}$. Baena

Unidad de Saneamiento y Biotecnología Ambiental, Departamento de Biología, Pontificia Universidad Javeriana,

POB 56710, Bogotá, DC, Colombia

V. Klepac-Ceraj $\cdot$ R. Kolter

Harvard Medical School,

200 Longwood Avenue,

Boston, MA 02115, USA

Present Address:

V. Klepac-Ceraj

The Forsyth Institute,

245 First Street,

Cambridge, MA 02142, USA 